\title{
The Unconscious Experimentally Demonstrated by Means of Hypnosis
}

\author{
Edoardo Casiglia ${ }^{1,2 *}$, Valérie Tikhonoff ${ }^{1,2}$, Enrico Facco ${ }^{2,3}$ \\ ${ }^{1}$ Department of Medicine, University of Padua, Padua, Italy \\ ${ }^{2}$ Institute Franco Granone, Italian Centre for Clinical and Experimental Hypnosis, Turin, Italy \\ ${ }^{3}$ Studium Patavinum, University of Padua, Padua, Italy \\ Email: "edoardo.casiglia@unipd.it
}

Received 26 February 2016; accepted 10 April 2016; published 13 April 2016

Copyright (C) 2016 by authors and Scientific Research Publishing Inc.

This work is licensed under the Creative Commons Attribution International License (CC BY). http://creativecommons.org/licenses/by/4.0/

(c) (;) Open Access

\section{Abstract}

Although taken for granted today by people and by some experts, the unconscious has never been experimentally demonstrated. Even for the psychoanalysts, the unconscious is nothing more than a model. The unconscious, if anything, is normally obscured by conscious activities and can only express itself in response to conditions leading to non-ordinary mental expressions, for instance during hypnosis. For many years, we have been using hypnosis in variegating experimental setting, and we think one of the evidences coming from our tests is the experimental demonstration that the unconscious exists and can be forced to respond to solicitations the participant is not aware of. We administered hypnotic suggestions to highly-hypnotizable normal participants with the aim of inducing hallucination of body heating, alexia, amusia, spatial neglect, focused analgesia, general anaesthesia, and age regression. Following such suggestions, participants actually experienced a sensation of heat, incapability to read, lack of interest in a side of the world, indifference to painful stimuli, and revivification of infantile age, respectively. But this is not all. Through the above-mentioned suggestions we also obtained some physical reactions that could only be defined as unconscious, i.e. increase of the stroke volume and of the mesenteric artery flow following hallucinated body heating, reduction of reaction times to incongruent color words in a Stroop task following alexia, prolongation of ipsilateral reaction times following spatial neglect, reduction of mismatch negativity to deviant stimuli following hypnotic amusia, coherent modifications of the sympathetic/parasympathetic balance to trigeminal and non-trigeminal pain during analgesia and anaesthesia, reduction of Raven score and Raven-induced stress during age regression. These responses evoked during hypnosis in response to mental images are clearly non-voluntary and non-conscious, and demonstrate in experimental setting with the tools that are typical of human physiology-the existence of unconscious to perceive and react.

"Corresponding author. 
Keywords

Haemodynamics, Regressive, Analgesia, Unconscious, Stroop, Hallucination, Neglect

\section{Introduction: The Unconscious-Creed or Evidence?}

The existence of the unconscious is taken for granted by common people. Psychoanalysis gave a great impulse to the belief that the unconscious exists. And the concept that the psychoanalytic rapport opens a window onto the unconscious is generally accepted today. And ultimately, if psychoanalysis works, the unconscious, as a matter of fact, must be a reality. In other words, there is an evident validity of the effects of the unconscious that should give witness to its existence.

Nevertheless, the unconscious remains quite elusive.

It must be emphasized that the unconscious is, even according to Freud, nothing more than a model, useful to explain some phenomena such as start of action, neuroses, psychopathology of everyday life, artistic sublimation and psychosomatic diseases. But all the attempts to find an anatomic basis for the unconscious have failed, and the current belief is that it is appropriate to abstain from considering it as a real space. It is also plausible that the unconscious is simply an aspect of mind activity, not easily or positively detachable from consciousness, or that the consciousness and the unconscious constitute a continuum made of interchangeable information.

Furthermore, a fringe of physicians de facto doubts of the existence of the unconscious, or disregards it: up to, the unconscious has therefore the characteristics of a creed rather than of a scientific reality. Thus, there is longing and urgent need of its experimental demonstration.

Furthermore, many psychologists and psychotherapists concretely think the unconscious has no practical role in physical world. On the contrary, it is important to underline that the unconscious acts give not only psychic but also physical products. If psychosomatic medicine exists, it must be because the unconscious exists in turn. Now, although everyone is ready to acknowledge that psychosomatic medicine has the dignity of a concrete discipline, no one has tried to demonstrate in experimental setting the concreteness of the unconscious.

The unconscious, if anything, is normally dominated and obscured by conscious activities and can only express itself when consciousness is depressed because of an abaissement du niveau mental. This happens spontaneously or in response to pharmacological or non-pharmacological procedures leading to non-ordinary mental expressions, for instance following hypnosis.

\section{Hypnosis as an Experimental Tool}

Through hypnotism (induction to hypnosis), non-conscious effects having the characteristics of reality can be obtained in response to a mental image (Casiglia, 2008, 2012). It must be clarified that a mental image becoming plastic (Barnier \& McConkey, 1999) is the crucial point in experimental hypnosis aimed at demonstrating the unconscious, as it guarantees that the effects observed in experimental setting, if any, are not mere automatic neurovegetative responses, but the consequence of the superior mental activity of Homo sapiens.

The medium producing such responses (a medium that is non-conscious) must have, in turn, the characteristics of reality. This unconscious activity and this unconscious function are the links between hypnosis and its consequences in the real world. Hypnosis is therefore a facilitator, a catalysing agent making possible "beyond the mirror" what would be impossible in the real world. Its avail is that its results are perfectly valid in the real world.

According to this view, a limited number of authors are using hypnotism as a tool to study experimentally some aspects of human mind. For instance, it has recently been demonstrated with imaging techniques that hypnotic paralysis and arm levitation following hypnotic suggestions are real and are accompanied by (or are perhaps produced by) a modified representation of the self impacting motor abilities (Burgmer et al., 2013; Cojan et al., 2009); the precuneus could play a pivotal role in producing (Casiglia et al., 2012a) and maintaining (Pyka et al., 2011) the particular mind state called hypnosis, making possible unconscious motor modifications.

Motor tasks apply well to neurophysiological studies, but our opinion is that voluntary motor activities are not the best target when one wants to demonstrate the unconscious exists. To this aim, effects that are notoriously independent of will and of voluntary control should be chosen. Furthermore, we think neuroimaging techniques 
are not the best way as they are static and not adapt to put in evidence the dynamic, variable, unstable, functional entity represented by the unconscious.

Only few researchers are admittedly using hypnotism as a tool to explore or demonstrate the unconscious. For instance, hypnotic amnesia has been used to demonstrate that hypnosis has little effect on the retrieval of the proscribed information, being the retrieved information blocked from conscious processing consistently with the operation sometimes called non-conscious free will (Glaser \& Kihlstrom, 2005; Haggard et al., 2004; Smith et al., 2013).

In our laboratory of experimental hypnosis, the goal to analyze some particular aspects of the unconscious with the aim to demonstrate indirectly its existence is pursued. Neuroimaging (Casiglia et al., 2010, 2012; Priftis et al., 2011) is not the only tool used by our research group, being our research activity also based on functional tests and on cardiovascular measurements.

\section{Demonstrating the Unconscious by Means of Hypnosis}

\subsection{Important Premises}

During the last years we used hypnotism to demonstrate that the unconscious exists and can be driven to react to solicitations independent of participant's awareness. Hypnotism is a very useful experimental tool for physiologists as the effects of plastic monoideims are experienced as real and have physical consequences that can be repeated and measured. Actually, our research group is particularly interested in clinically measurable, repeatable, physical consequences of hypnosis (Casiglia et al., 2012), and performed a coordinated set of experiments on this topic (Casiglia, 2012; Casiglia et al., 1997, 2006, 2007, 2010, 2012, 2012a; Facco et al., 2011, 2014; Giordano et al., 2012; Priftis et al., 2011; Tikhonoff et al., 2012).

To use hypnotism with the aims described above, it is necessary to build preliminarily appropriate experimental models. In this modeling, an hypothesis is proposed regarding an objective (for instance, demonstrating the existence of the unconscious), and a procedure based on hypnotism is arranged to confirm or deny the hypothesis: induction of hypnosis is followed by a phase of neutral hypnosis, then a mental image potentially able to induce a physical modification that is notoriously out of voluntary control is suggested.

This stringent modeling is absolutely necessary in order to be sure that the physical modifications observed, if any, are the consequence of an unconscious mental image (typical of Homo sapiens) and not of an automatic response of the neurovegetative system. For instance, if an elderly men passing from the supine to the standing position experiences a blood pressure fall due to baroceptor malfunction, this is an automatic neurovegetative response, if he experiences the same blood pressure fall while lying, thinking to be standing, this is not an automatic response but the physical consequence of a mental image. Thanks to wide literature, it is clear today that "the human mind is capable of maintaining unconscious vigilance over its own automatic processes", suggesting "a volitional nature of the unconscious" (Glaser \& Kihlstrom, 2005). This is the setting in which our experiments are performed.

The research activity on this topic is still current in our laboratory while we are writing the present paper, and new experimental models are continuously conceived and tested. In the next paragraphs, 6 of these models are described.

\subsection{Model 1: Hypnotic Body Heating}

The hypothesis that the unconscious exists was first evaluated by our research group by testing the possibility to obtain during hallucination of body heating an increase in stroke volume (SV), i.e. in the amount of blood ejected from left ventricle at each systole. The sequence of experimental events is hypnotic induction $\rightarrow$ suggestion of body heating $\rightarrow$ mental image of body heating becoming plastic $\rightarrow$ measurement of SV variations if any. The idea is that, if SV increases in response to the hypnotically-induced mental image of body heating, the unconscious must exist, as there is no conscious way to increase SV voluntarily.

When we applied this model to 18 highly-hypnotizable participants receiving an hypnotic suggestion of warm tub bathing (Figure 1), peripheral, thoracic and splanchnic vasodilation was observed, as demonstrated by the increase in forearm flow $(+43 \%, p<0.01)$, cardiac index $(+28 \%, p<0.01)$ and mesenteric artery flow $(+59 \%, p$ $<0.01$ ), a behavior similar to that observed in real body heating (Casiglia et al., 2006). Cardiac output rise was due to $28 \%$ increase $(p<0.01)$ in stroke volume (Figure 2$)$. Mesenteric artery diameter even tended to increase by $16 \%$ (Casiglia et al., 2015, 2015a). 


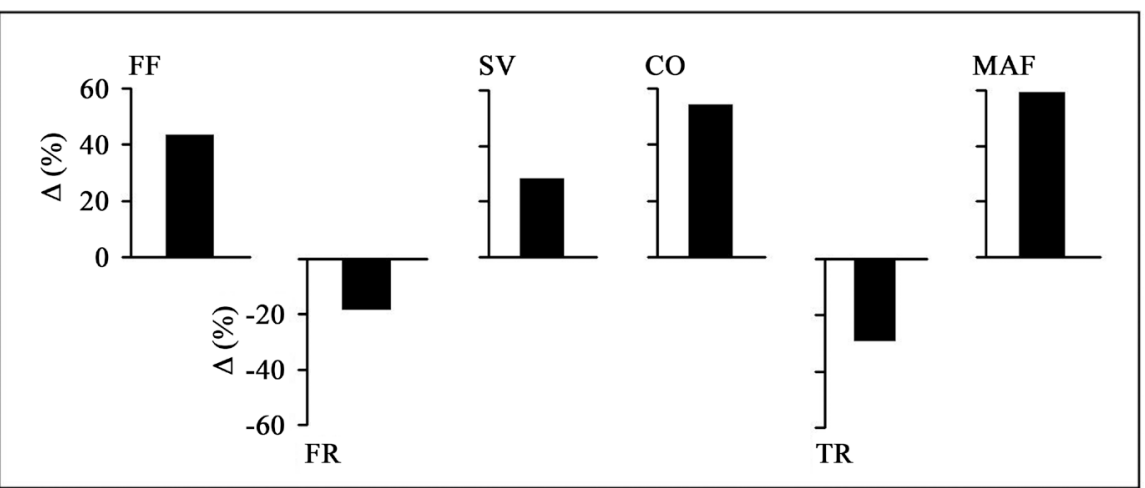

Figure 1. Hypnotic body heating. Percent variations $(\Delta)$ of hemodynamic parameters following the non-ordinary mental expression called "hypnotic hallucination of warm tub bathing". Forearm flow (FF) increases by $43 \%$, stroke volume (SV) by $28 \%$, cardiac output (CO) by $54 \%$ and mesenteric artery flow (MAF) by $59 \%$. Forearm resistance (FR, $-18 \%$ ) and total resistance $(\mathrm{TR},-29 \%)$ mirror flows increase as in real immersion in warm water. All variations are statistically significant vs. non-hypnotic baseline conditions $(p<0.05)$ (Casiglia et al., 2006; and personal unpublished data by Tikhonoff, Senzolo and Casiglia, 2012). This unconscious response to hypnotic body heating demonstrates the unconscious exists.

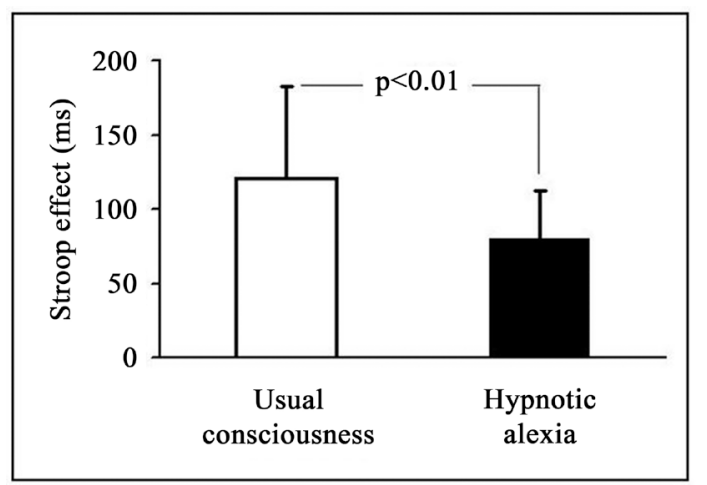

Figure 2. Hypnotic neglect for written language. Color words are used in setting of Stroop task. Incongruence between color and color word creates a Stroop effect that is in average $120 \mathrm{~ms}$ in conditions of usual consciousness ( $\square$ ). During hypnotic alexia ( $\mathbf{a})$ the Stroop effect is significantly reduced by $34 \%(p<0.01)$, indicating the metaphor of written language is lost (Casiglia et al., 2010). This unconscious response to hypnotic alexia demonstrates the unconscious exists.

As the participants knew the effects of heating on human body because of their own previous everyday experiences of tub bathing, one could suspect that systemic vasodilation in response to mental image of body heating was at the limit a conscious phenomenon. On the contrary, SV could only increase unconsciously, not voluntarily, as (apart from some physiologists and cardiologists) people are not aware that SV is a determinant of cardiac output and increases in body heating. As a consequence, it is evident that something acting as what we normally call "the unconscious" worked in our experiment, not only dilating peripheral and abdominal vessels, but also increasing heart contractility, independent of consciousness and will, in response to mental image of warm tub bathing. Mental image must be the cause of these variations, as nothing else changed during the experiment.

Interestingly, portal vein diameter decreased by $25 \%(p<0.01)$ in the above-mentioned experiment (Casiglia et al., 2015, 2015a). This finding, probably representing a compensatory mechanism, was unexpected because the portal response to body heating is practically unknown. This unconscious response was therefore independent of the consciousness of the participants and even of the consciousness of the researchers (a sort of double-blind unconscious experiment).

\subsection{Model 2: Hypnotic Neglect for Language}

In another experiment we demonstrated the existence of the unconscious using reaction times (RT) to visual stimuli as a marker. The idea was to abolish via hypnotic suggestions the metaphor of language, producing what 
we called hypnotic alexia (Casiglia et al., 2010), and then to measure the response to a Stroop task using color words (Stroop, 1935). The sequence of experimental events was hypnotic induction $\rightarrow$ suggestion of alexia $\rightarrow$ mental image of alexia becoming plastic $\rightarrow$ Stroop task $\rightarrow$ measurement of variations in RT if any. The rationale of this model was that: 1) cognitive processes can be unconsciously or voluntarily controlled (Shiffrin et al., 1997); 2) unconsciously-activated non-voluntary processes cannot be easily interrupted; and 3) words are processed unconsciously (Mac Leod, 1991). The principle postulated by Stroop is that reading color words interferes with word color, reducing reactivity and consequently slowing down the identification of words written in interfering color (incongruence) in comparison to congruence.

In practical terms, in this model we asked 12 healthy highly-hypnotizable participants ( 6 males and 6 females aged 28.6 \pm 3.8 years recruited among the medical and psychological university staff whose attitude to hypnosis was previously ascertained by means of Stanford Hypnotic Susceptibility Scale Form C in order to define hypnotizability, and by means of Minnesota Multiphasic Personality Inventory in order to exclude borderline subjects at risk of psychotic decompnsation), to detect, by pressing a button, the color of some color words (green, red, yellow, blue) appearing on a screen in congruent or incongruent colors, paying no conscious attention to their verbal meaning (but, of course, unconscious attention was paid in spite of our request). Well, in case of incongruence, RT were $120 \mathrm{~ms}$ longer $(p<0.01)$ than in case of congruence (i.e., the Stroop effect was $120 \mathrm{~ms}$ ). After hypnotic alexia was produced, the Stroop effect was halved (Casiglia et al., 2010) (Figure 2). As no conscious, voluntary control is possible in this range of RT, something of unconscious must have acted in this experiment after hypnotic alexia was reached: the hypnotic mental image of alexia evidently produced an unconscious neglect for written language leading to greater unconscious congruence when colors were conflicting with color words, so demonstrating the existence of the unconscious.

Interestingly, RT were reduced following hypnotic alexia in highly-hypnotizable participants only, not in scarcely-hypnotizable ones, and a significant interaction between suggestion, congruence and hypnotizability was detected $(\mathrm{F}=5.33, p<0.04)$. This confirms that hypnosis was really the cause of hypnotic alexia.

\subsection{Model 3: Hypnotic Neglect for Rhythm}

Our research group then decided to induce neglect for rhythm in 10 healthy volunteers by measuring mismatch negativity (MMN) before and during a neglect that we called hypnotic amusia for rhythm (Facco et al., 2014). The sequence of experimental events was hypnotic induction $\rightarrow$ suggestion of amusia $\rightarrow$ mental image of amusia becoming plastic $\rightarrow$ administration of deviant stimuli $\rightarrow$ measurement of variations in MMN if any.

MMN is an auditory event-related potential generated by unconscious processes and widely distributed in the scalp with higher amplitude in right frontal areas (Alho, 1995; Naatanen, 1995). MMN is elicited by administration of deviant stimuli during a series of standard, predictable auditory stimuli, generating a neural mismatch between the deviant stimulus and the sensory memory trace yielded by standard stimuli. Therefore, MMN is involved in unconscious, non voluntary switch of attention toward a novel auditory stimulus, and can be recorded even when attention is focused elsewhere.

Basically, when participants received a deviant stimulus (e.g. beeeep in a series of beep), MMN was registered in his/her scalp. During amusia, the MMN amplitude decreased in average from $-8.2 \pm 2.2$ to $-5.4 \pm 1.1$ $\mu \mathrm{V}(-20 \%, p<0.05)$ in comparison to non-hypnotic conditions (Figure 3$)$. Of course this response to the mental image of hypnotic amusia must be unconscious, as there is no way to control either consciously or voluntarily the electroencephalographic tracings, particularly in the range of stimuli around $100 \mathrm{~ms}$.

This demonstrates once again the existence of the unconscious.

\subsection{Model 4: Hypnotic Spatial Neglect}

Spatial attention can be oriented by specific endogenous cues (Posner et al., 1982, 1987). Expert hypnotists can easily induce in highly hypnotizable subjects a condition characterized by hemilateral neglect. In experimental setting, we did this in 10 healthy volunteers (Priftis et al., 2011), and we believe the results demonstrated once again the existence of the unconscious. The sequence of experimental events was hypnotic induction $\rightarrow$ suggestion of hemilateral neglect $\rightarrow$ mental image of hemilateral neglect becoming plastic $\rightarrow$ administration of lateral visual stimuli $\rightarrow$ measurement of RT variation if any. In practical terms, in this experiment, RT to lateral visual stimuli were used before and after generating a neglect in which participants were made indifferent to the left or the right side of the world, alternatively: we reduced the participants' visuospatial attention either to the left or to 
the right visual hemifield, and asked them to react as fast as possible by pressing a button when becoming aware of a lateral visual stimulus on the screen.

In conditions of ordinary consciousness, no significant difference was recorded in response to stimuli coming from the left or the right (Figure 4). After reaching through appropriate hypnotic suggestions leading to mental image of left hemilateral neglect, RT to stimuli to the left visual hemifield were $145 \mathrm{~ms}$ longer $(p<0.05)$. In the same way, after reaching right hemilateral neglect, RT to stimuli to the right visual hemifield were $79 \mathrm{~ms}$ longer $(p<0.05)$.

Once again, this response could only be unconscious, as there is no way to control RT consciously or voluntarily in this range. And, of course, this had to be an unconscious response (pertaining to psychodynamics), not an automatic response to a brain lesion (pertaining to clinical neurology), as there was no lesion in this experimental setting with hypnotic suggestion of neglect.

Interestingly, we obtained the same results by inducing right "hyper-attention or left neglect", as well as "left hyper-attention or right neglect" (Priftis et al., 2011). In other words, directing visuospatial attention to the right visual hemifield caused unconscious left neglect, whereas directing visuospatial attention to the left hemifield caused unconscious right neglect, and viceversa. This is in agreement with the observations recorded by other authors in pathological conditions (Kinsbourne, 1993; Mark et al., 1988; Làdavas et al., 1993) and demonstrates the existence of an unconscious spatial attraction away from ipsilateral side, suggesting in turn that an unconscious hyper-attention to the counterlateral side is the crucial factor underlying the ipsilateral neglect. Neglect is therefore nothing more than an unconscious shift of attention from-left-to-right or from-right-to-left (Priftis et al., 2011).

All the above-mentioned results confirm the existence of the unconscious.

\subsection{Model 5: Analgesia}

Obtaining analgesia (even surgical analgesia) is amazingly easy in hypnosis. The best way is to suggest mental images leading to hypnotic focus analgesia (HFA), equivalent to pharmacological local anesthesia (Casiglia et al., 2007; Facco et al., 2011). In our experimental setting with HFA, the sequence of events was hypnotic induc-

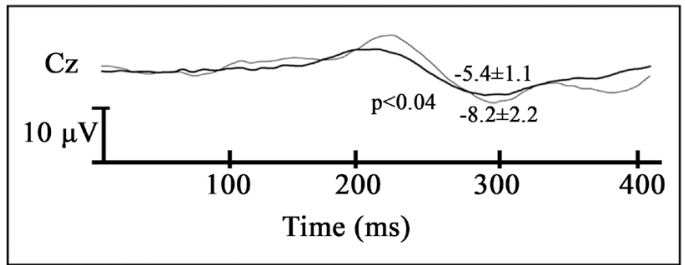

Figure 3. Hypnotic neglect for rhythm. Amplitude of mismatch negativity after autoditory stimuli from $\mathrm{Cz}$ at baseline. During the non ordinary mental experience amusia (heavy line) the mismatch negativity produced by an unexpected sound is reduced by $20 \%$ in comparison to baseline usual consciousness (thin lines) (Facco et al., 2014). This unconscious amusia demonstrates the unconscious exists.

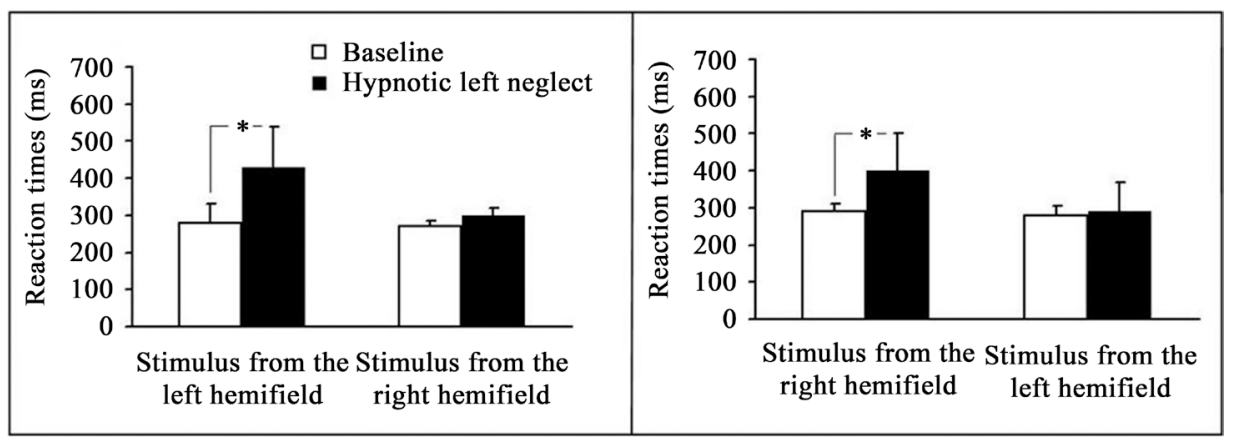

Figure 4. Hypnotic neglect for a side of the world. When neglect for the left hemifield ( $\square$ in upper panel) or for the right hemifield ( $\square$ in lower panel) is suggested, stimuli coming from the neglecting side are accompanied by $50 \%$ perception delay as measured with reaction times (Priftis et al., 2011). This unconscious response to the "hypnotic hemilateral neglect" demonstrates the unconscious exists. 
tion $\rightarrow$ suggestion of HFA $\rightarrow$ mental image of HFA becoming plastic $\rightarrow$ administration of a painful stimulus $\rightarrow$ measurement of pain perception, pain tolerance and haemodynamic reflex response if any.

In our experience, subjective perception of non-trigeminal pain decreased by $68 \%(p<0.01)$, and objective pain tolerance increased by $238 \%(p<0.01)$ when HFA was operating, i.e. when the participants underwent the hypnotic command to be analgesic in a certain non-trigeminal body segment (Casiglia et al., 2007). Also in the case of trigeminal electrical stimulation, the pain threshold increased by $220 \%(p<0.0001)$ (Facco et al., 2011).

What is really interesting is the cardiovascular response to pain when HFA was reached. As known, non-trigeminal (peripheral) pain is normally associated to increased sympathetic discharge with peripheral resistance rise (Casiglia et al., 2007), while trigeminal (dental) pain is accompanied by reduced peripheral resistance (Facco et al., 2011). This is what happened also in our experiment in conditions of usual consciousness (Figure 5, left panel). Nevertheless, this notion is not in the conceptual patrimony of non-professional people, being limited to a restricted number of physiologists, anaesthetists and cardiologists. Despite this, when HFA was suggested to normal people, the cardiovascular response to pain was always inhibited, leading to a reduced rise of resistance following non-trigeminal pain and to a reduced fall of resistance following trigeminal pain (Figure 5, right panel). As the participants did not know the difference between the cardiovascular consequences of the two types of pain, this different response to the mental image of HFA can only be unconscious, so demonstrating the existence of the unconscious.

More recently, we also explored in a pilot study performed in 9 healthy participants the possibility to induce total-body anaesthesia rather than HFA in the frame of what we called hypnotic general anesthesia. In this model, we induced all the modifications that are typical of spontaneous-breathing general anesthesia (analgesia + narcosis + amnesia + surgical immobility). The results obtained in this model were even more evident than those observed with HFA, and reflex cardiovascular response to non-trigeminal pain was reduced as well (Figure 5, right panel). As participants did not know where the experimental painful stimulus would be administered, hypnotic general anaesthesia must probably be considered even more effective and potent than HFA.

\subsection{Model 6: Age Regression}

Giving adequate hypnotic suggestions, it is relatively easy to induce in highly-hypnotizable subjects age regres-

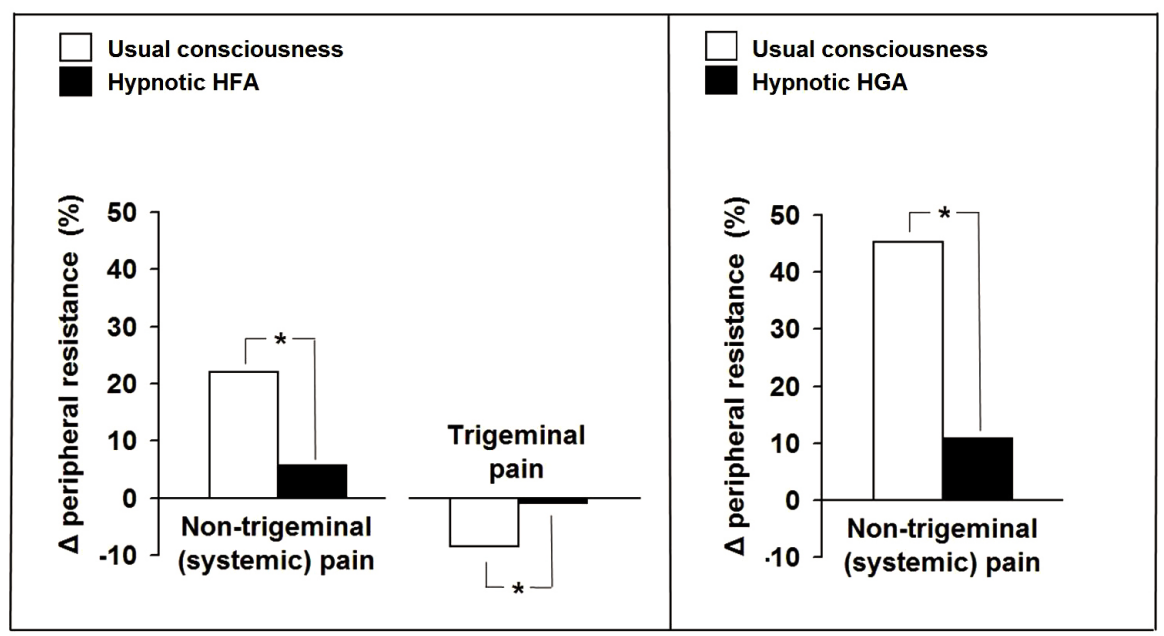

Figure 5. Hypnotic analgesia. In the left panel, "hypnotic analgesia" is produced (匹). The physiological reflex increase of peripheral resistance induced by non-trigeminal pain (hand in icy water) is significantly reduced by $74.5 \%$ in comparison to usual consciousness ( $\square$ ) during hypnotic focused analgesia (HFA), corresponding to local anaesthesia (Casiglia et al., 2007). The physiological reflex fall of peripheral resistance induced by trigeminal pain (electrical stimulation of right first premolar by a Digitest Serial D-22663 03/2005, Parkell Inc, Edgewood, USA) is also significantly reduced by $9 \%$ during local HFA (Facco et al., 2011). In the right panel, the physiological reflex increase of peripheral induced by non-trigeminal pain (electrical stimulation of the median nerve) is significantly reduced by $76.3 \%$ during normal-breathing hypnotic general anesthesia (HGA), characterized by total body analgesia, peripheral paralysis, narcosis and amnesia ( $\mathbf{})$, in comparison to usual consciousness ( $\square$ ) (Casiglia et al., unpublished data). These three unconscious responses to analgesia demonstrate the unconscious exists. 
sion and revivification (ARAR). In such conditions, participants not only remember episodes of their adolescence or childhood, but also experience them once again, being completely absorbed by the past. We used this model too to demonstrate the existence of the unconscious. The sequence of the experimental events was hypnotic induction $\rightarrow$ suggestion of ARAR $\rightarrow$ mental image of ARAR becoming plastic $\rightarrow$ quantification of variations in Rorshach test (for age-specific personality) and Raven test (for age-specific intelligence) if any.

Before hypnotic induction, in conditions of usual consciousness, 10 healthy participants underwent a Rorschach test and a Raven test to ascertain their personality and intelligence, respectively. Regression to the age of 6 years was chosen for this experiment (Giordano et al., 2012). After ARAR was reached, the tests were repeated (this was not easy, as participants showed childlike behavior with disorientation and weeping, often crying and asked for mum, etc.). The Rorschach testified for an infantile personality, very different from the adult one. This was demonstrated by $129 \%$ increase $(p<0.01)$ of "detail" localizations and $24 \%$ reduction $(p<0.01)$ of "global" localizations in comparison to basal conditions (Giordano et al., 2012) (Figure 6). Not only this, but the Raven score, that was on average $50.3 \pm 4.3$ at the anagraphic age and did not change in neutral hypnosis,

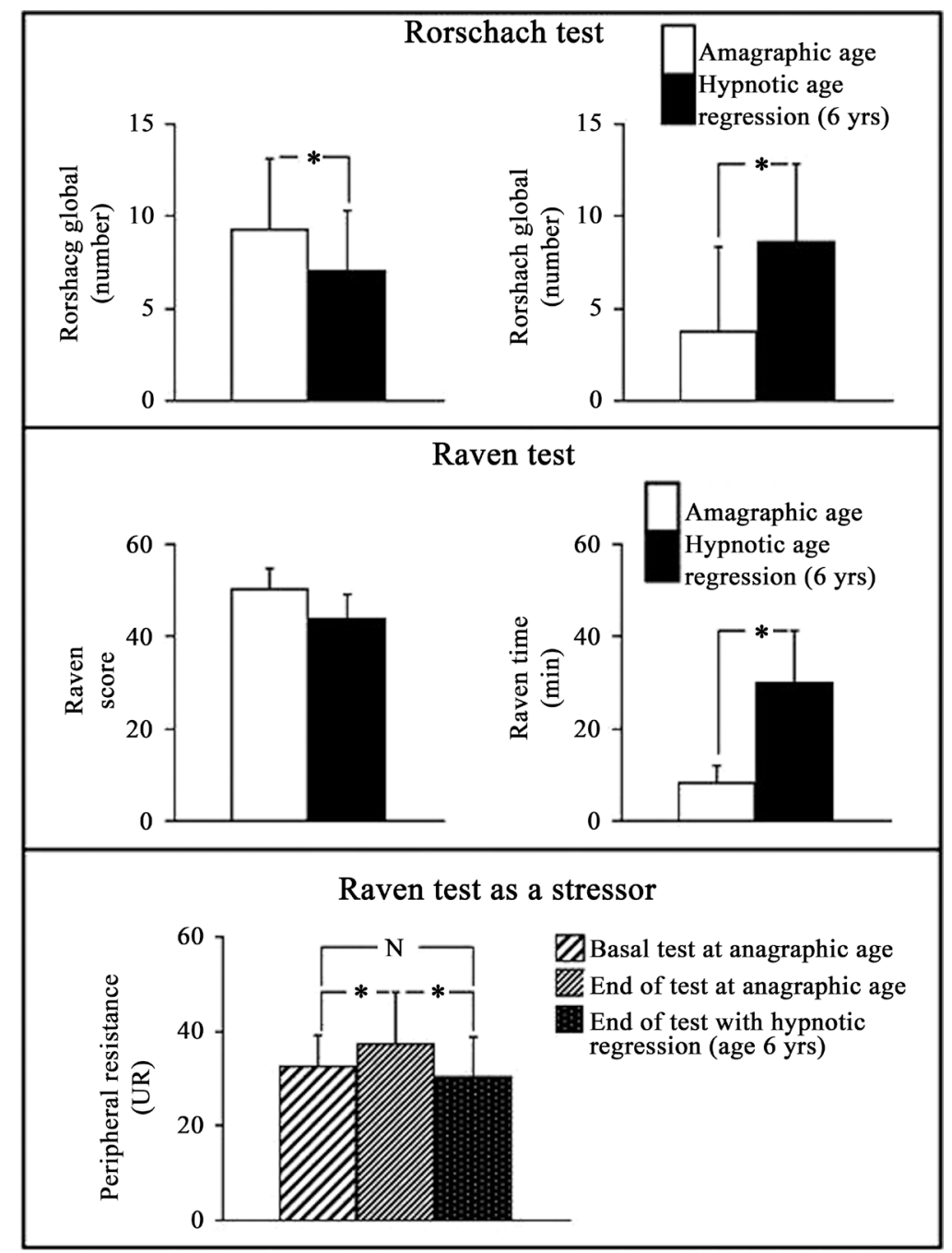

Figure 6. Hypnotic age regression and revivification. In upper panels, "detail" responses to the Rorschach test significantly increase while global responses decrease in hypnotic age regression ( $\mathbf{\square})$ in comparison to anagraphic age ( $\square$ ), indicating infantile personality. In intermediate panels, the performance at Raven test is significantly reduced and the time to complete the task is significantly increased during age regression. In lower panels, the reflex increase of peripheral resistance in response to the Raven test considered as a stressor is significantly lower during age regression than in basal non-hypnotic conditions (and non significantly different from the baseline), indicating lower level of stress as in a playful approach to the test (Giordano et al., 2012). These unconscious responses demonstrate the unconscious exists. 
decreased by $12.5 \%$, and the time necessary to complete the test increased by $85.5 \%$ in ARAR (Figure 6).

This is per se very striking, as common people cannot control the response to Rorschach or Raven test simulating to be younger (this could perhaps be a prerogative of an expert psychologist). But what is really demonstrative of the existence of the unconscious is the cardiovascular response to the Raven test as a stressor. In basal conditions, the administration of the Raven matrices was accompanied, as expected, by a significant increase of peripheral resistance with reduction of SV due to reflex post-load fall (Figure 6). A comparable behavior was observed when the test was administered in neutral hypnosis at real age. Conversely, when the test was administered in hypnotic ARAR at the age of 6 years, no significant variation of the above-mentioned cardiovascular parameters was observed. We interpret these finding by arguing that a playful approach, typical of childhood, deprived the Raven test of its stressor effect, so reducing its impact on the sympathetic discharge.

These objective variations of the cardiovascular response to the test (which are obviously non-voluntary and cannot be simulated) confirm the existence of the unconscious.

\section{Conclusion}

From the models described above, the unconscious clearly appears to be existent for the explicit reason that it is the only force able to produce some effects. This force emerging from our experiments is out of any conscious, voluntary control; it is blind and totipotent; it can have all the trappings of a physiological agent (like in psychosomatic disorders) and can be demonstrated in an experimental setting with the tools and the devices that are typical of Galileans sciences such as physiology and cardiology. More importantly, it must not be confounded with an automatic neurovegetative response, as it is evoked by a mental image (not by a mere reflex) and acts through the superior function of plastic monoideism.

After demonstrating the unconscious exists, the possibility is now open to characterize better its nature. A possibility is to combine the methods and the results of physiological research described herein to those that are typical of neuroimaging, trying to give a neurological substratum to unconscious non-voluntary responses. Functional magnetic resonance imaging is the best candidate to do this, and our group has in the pipeline a series of experiments with this methodology.

The present paper is a pilot article. Its limitations are represented by the relatively low number of participants in each model, and by the fact that different models are examined in different times and over different participants. Coordinated research repeating the same experiments in a large and homogenous group of subjects is desirable.

\section{References}

Alho, K. (1995). Cerebral Generators of Mismatch Negativity (MMN) and Its Magnetic Counterpart (MMNm) Elicited by Sound Changes. Ear and Hearing, 16, 38-51. http://dx.doi.org/10.1097/00003446-199502000-00004

Barnier, A. J., \& McConkey, K. M. (1999). Hypnotic and Posthypnotic Suggestion: Finding Meaning in the Message of the Hypnotist. International Journal of Clinical and Experimental Hypnosis, 47, 192-208. http://dx.doi.org/10.1080/00207149908410032

Burgmer, M., Kugel, H., Pfleiderer, B., Ewert, A., Lenzen, T., Piocha, R., Pykac, M., Sommerd, J., Aroltc, V., Heufta, G., \& Konrad, C. (2013). The Mirror Neuron System under Hypnosis-Brain Substrates of Voluntary and Involuntary Motor Activation in Hypnotic Paralysis. Cortex, 49, 437-445. http://dx.doi.org/10.1016/j.cortex.2012.05.023

Casiglia, E. (2008). Hypnosis in the Theory of the Bicameral Mind. The Jaynesian, 2, 12-14.

Casiglia, E. (2012). Why Is It So Easy to Hypnotize? Contemporary Hypnosis and Integrative Therapy, 29, 309-314.

Casiglia, E., Mazza, A., Ginocchio, G., Onesto, C., Pessina, A. C., Rossi, A., Cavatton, G., \& Marotti, A. (1997). Haemodynamics Following Real and Hypnosis-Simulated Phlebotomy. Americam Journal of Clinical Hypnosis, 4, 368-375. http://dx.doi.org/10.1080/00029157.1997.10403405

Casiglia, E., Rossi, A., Tikhonoff, V., Scarpa, R., Tibaldeschi, G., Giacomello, M., Cannaa, P., Schiavona, L., Rizzatoa, A., \& Lapentab, A. M. (2006). Local and Systemic Vasodilation Following Hypnotic Suggestion of Warm Tub Bathing. International Journal of Psychophysiology, 62, 60-65. http://dx.doi.org/10.1016/j.ijpsycho.2006.01.012

Casiglia, E., Schiavon, L., Tikhonoff, V., Haxhi Nasto, H., Azzi, M., Rempelou, P. et al. (2007). Hypnosis Prevents the Cardiovascular Response to Cold Pressor Test. American Journal of Clinical Hypnosis, 49, 255-266.

http://dx.doi.org/10.1080/00029157.2007.10524503

Casiglia, E., Schiff, S., Tikhonoff, V., Schiavon, L., Bascelli, A., Nasto, H. H., Guidotti, F., Giacomello, M., \& Amodio, P. 
(2010). Neurophysiological Correlates of Post-Hypnotic Alexia: A Controlled Study with Stroop Test. American Journal of Clinical Hypnosis, 52, 219-233. http://dx.doi.org/10.1080/00029157.2010.10401721

Casiglia, E., Tikhonoff, V., \& Rossi, A. M. (2015a). Allucinazioni Positive. In E. Casiglia (Ed.), Trattato d'Ipnosi e Altre Modificazioni di Coscienza (pp. 593-601). Padova: CLEUP.

Casiglia, E., Tikhonoff, V., Giordano, N., Regaldo, G., Facco, E., Marchetti, P. et al. (2012a). Relaxation versus Fractionation as Hypnotic Deepening: Do They Differ in Physiological Changes? International Journal of Clinical and Experimental Hypnosis, 60, 338-355. http://dx.doi.org/10.1080/00207144.2012.675297

Casiglia, E., Tikhonoff, V., Giordano, N., Regaldo, G., Tosello, M. T., Rossi, A. M., Bordin, D., \& Facco, M. G. E. (2012). Measured Outcomes with Hypnosis as an Experimental Tool in a Cardiovascular Physiology Laboratory. International Journal of Clinical and Experimental Hypnosis, 60, 241-261. http://dx.doi.org/10.1080/00207144.2012.648078

Casiglia, E., Tikhonoff, V., Miroglio, G., Giordano, N., Boschetti, G., Guidotti, F. et al. (2015). Notazioni Generali sui Modelli e sulla Tecnologia. In E. Casiglia (Ed.), Trattato d'Ipnosi e Altre Modificazioni di Coscienza (pp. 567-583). Padova: CLEUP.

Cojan, Y., Waber, L., Schwartz, S., Rossier, L., Forster, A., \& Vuilleumier, P. (2009). The Brain under Self-Control: Modulation of Inhibitory and Monitoring Cortical Networks during Hypnotic Paralysis. Neuron, 62, 862-875. http://dx.doi.org/10.1016/j.neuron.2009.05.021

Facco, E., Casiglia, E., Masiero, S., Tikhonoff, V., Giacomello, G., \& Zanette, G. (2011). Effects of Hypnosis on Dental Pain Threshold. International Journal of Clinical and Experimental Hypnosis, 59, 454-468. http://dx.doi.org/10.1080/00207144.2011.594749

Facco, E., Ermani, M., Rampazzo, P., Tikhonoff, V., Saladini, M., Zanette, G., Casiglia, E., \& Spiegel, D. (2014). Top-Down Regulation of Left Temporal Cortex by Hypnotic Amusia for Rhythm: A Pilot Study on Mismatch Negativity. International Journal of Clinical and Experimental Hypnosis, 62, 129-144. http://dx.doi.org/10.1080/00207144.2014.869124

Giordano, N., Tikhonoff, V., Tosello, M. T., Lapenta, A. M., \& Casiglia, E. (2012). An Experimental Approach to Hypnotic Age Regression: Controlled Study over 10 Healthy Participants. Contemporary Hypnosis and Integrative Therapy, 29, 271-283.

Glaser, J., \& Kihlstrom, J. F. (2005). Compensatory Automaticity: Unconscious Volition Is Not an Oxymoron. In R. R. Hassin, J. S. Uleman, \& J. A. Bargh (Eds.), The New Unconscious (pp. 171-195). Oxford Series in Social Cognition and Social Neuroscience. Oxford: Oxford University Press.

Haggard, P., Cartledge, P., Dafydd, M., \& Oakley, D. A. (2004). Anomalous Control: When Free-Will Is Not Conscious. Consciousness and Cognition, 13, 646-654. http://dx.doi.org/10.1016/j.concog.2004.06.001

Kinsbourne, M. (1993). Interactive Attentional Systems and Visual Neglect. In G. W. Humphreys, \& M. J. Riddoch (Eds.), Unilateral Neglect: Clinical and Experimental Studies (pp. 63-86). Howe: Lawrence Erlbaum.

Làdavas, E., Umiltà, C., Ziani, P., Brogi, A., \& Minarini, M. (1993). The Role of Right Side Objects in Left Side Neglect: A Dissociation between Perceptual and Directional Motor Neglect. Neuropsychologia, 31, 761-773. http://dx.doi.org/10.1016/0028-3932(93)90127-L

Mac Leod, C. M. (1991). Half a Century of Research on the Stroop Effect: An Integrative Review. Psychological Bulletin, 109, 163-203. http://dx.doi.org/10.1037/0033-2909.109.2.163

Mark, V. W., Kooistra, C. A., \& Heilman, K. M. (1988). Hemispatial Neglect Affected by Non-Neglected Stimuli. Neurology, 38, 1207-1211. http://dx.doi.org/10.1212/WNL.38.8.1207

Naatanen, R. (1995). The Mismatch Negativity: A Powerful Tool for Cognitive Neuroscience. Ear and Hearing, 16, 6-18. http://dx.doi.org/10.1097/00003446-199502000-00002

Posner, M. I., Cohen, Y., \& Rafal, R. D. (1982). Neural Systems Control of Spatial Orienting. Philosophical Transactions of the Royal Society B: Biological Sciences, 298, 187-198. http://dx.doi.org/10.1098/rstb.1982.0081

Posner, M. I., Walker, J. A., Friedrich, F. A., \& Rafal, R. D. (1987). How Do the Parietal Lobes Direct Covert Attention? Neuropsychologia, 25, 135-145. http://dx.doi.org/10.1016/0028-3932(87)90049-2

Priftis, K., Schiff, S., Tikhonoff, V., Giordano, N., Amodio, P., Umiltà, C., \& Casiglia, E. (2011). Hypnosis Meets Neurosciences: Simulating Visuospatial Neglect in Healthy Participants. Neuropsychologia, 49, 3346-3350. http://dx.doi.org/10.1016/j.neuropsychologia.2011.08.008

Pyka, M., Burgmer, M., Lenzen, T., Pioch, R., Dannlowski, U., Pfleiderer, B. et al. (2011). Brain Correlates of Hypnotic Paralysis: A Resting-State fMRI Study. Neuroimage, 56, 2173-2182. http://dx.doi.org/10.1016/j.neuroimage.2011.03.078

Shiffrin, R. M., \& Schneider, W. (1997). Controlled and Automatic Human Information Processing. Psychological Revue, $84,1-66$

Smith, C. H., Oakley, D. A., \& Morton, J. (2013). Increased Response Time of Primed Associates Following an Episodic Hypnotic Amnesia Suggestion: A Case of Unconscious Volition. Consciousness and Cognition, 22, 1305-1317. http://dx.doi.org/10.1016/j.concog.2013.08.003 
Stroop, J. P. (1935). Studies of Interference in Serial Verbal Reactions. Journal of Experimental Psychology, 18, 643-661. http://dx.doi.org/10.1037/h0054651

Tikhonoff, V., Azzi, D., Boschetti, G., Giordano, N., Rempelou, P., Giacomello, G. et al. (2012). Increase of Isometric Performance via Hypnotic Suggestion: Experimental Study over 10 Young Healthy Volunteers. Contemporary Hypnosis and Integrative Therapy, 29, 352-362. 